\title{
LA HUELLA URBANA DE LA FIRMA ROBLEDO HERMANOS EN LA CIUDAD DE BUCARAMANGA*
}

Néstor José Rueda Gómez***

Universidad Santo Tomás (Colombia)

Recibido: 20 junio 2012

Aprobado: 7 septiembre 2012

Miembros de la Familia Robledo en 1960 En la imagen: Elías, sentado a la derecha; de pie: Emilio, Fabio, Iván, Silvio y Dago Robledo, Sentado a la Izquierda don Emilio Robledo. padre, a sus 85 años. Fuente: Vanguardia Liberal, Bucaramanga, I de octubre de 1960, copic suministrada por el autor

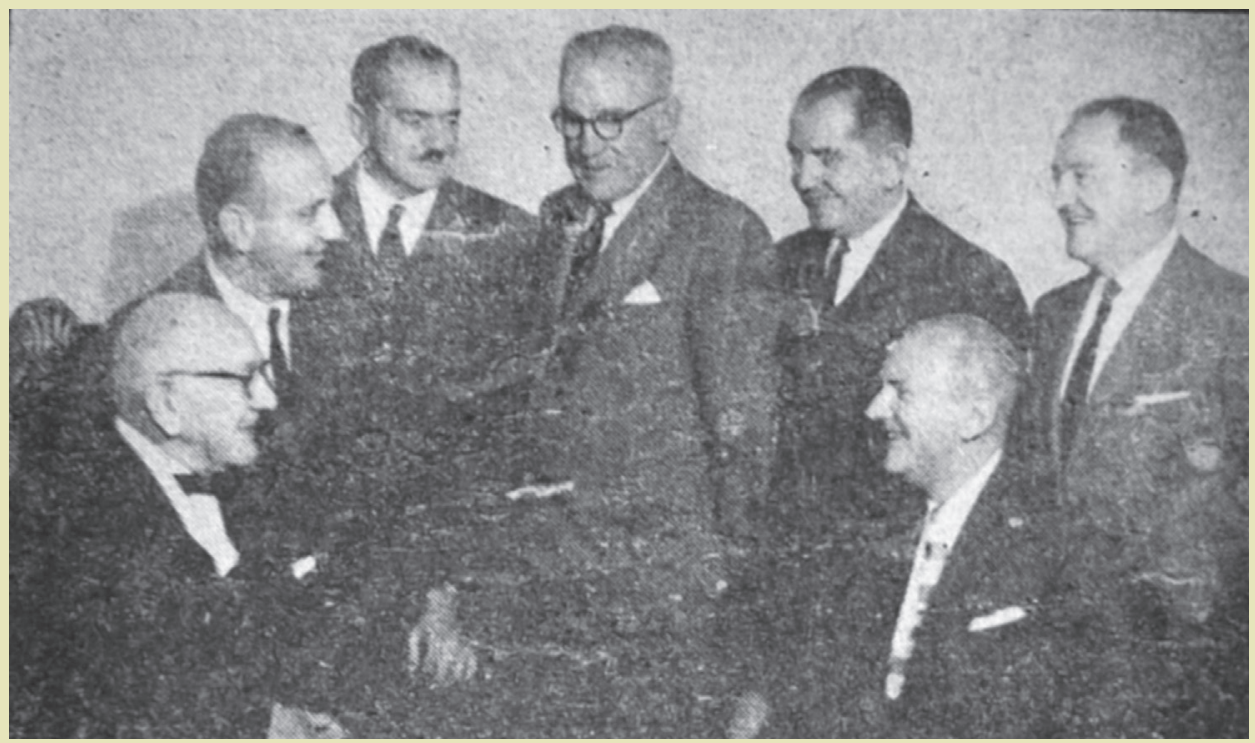

\section{RESUMEN}

El presente artículo es resultado de trabajo de investigación "Los actores de la vivienda privada en el Área Metropolitana de Bucaramanga: el caso Robledo Hermanos Ltda." Aprobado por la V Convocatoria Interna de Investigaciones, de la Universidad Santo Tomás de Bucaramanga, Colombia.

** Historiador Universidad Industrial de Santander, Doctor en Régimen Jurídico y Ordenamiento Urbano de la Universidad Politécnica de Valencia UPV, España. Líder del Grupo de Investigaciones de la Facultad de Arquitectura de la Universidad Santo Tomás de Bucaramanga, Colombia. nergoz80@yahoo.es

En el presente artículo se muestra el impacto que produjo la firma Robledo Hermanos, en el proceso urbanizador de la ciudad de Bucaramanga entre las décadas del cuarenta al setenta del siglo XX. El artículo está estructurado de la siguiente forma: una introducción, en la que se describe brevemente la forma como la familia Robledo se afincó en Bucaramanga e inició su periplo urbanizador, acompañado de una sucinta contextualización histórica. Luego se describen algunos de los resultados de síntesis de las actuaciones urbanas desarrolladas por la firma a lo largo de treinta años. Finalmente se presentan algunas conclusiones que podrían dar pie a futuras investigaciones.

\section{PALABRAS CLAVE}

Bucaramanga, Robledo Hermanos, urbanización, actores urbanos. 


\section{ROBLEDO HERMANOS URBAN FOOTPRINT IN THE CITY OF BUCARAMANGA}

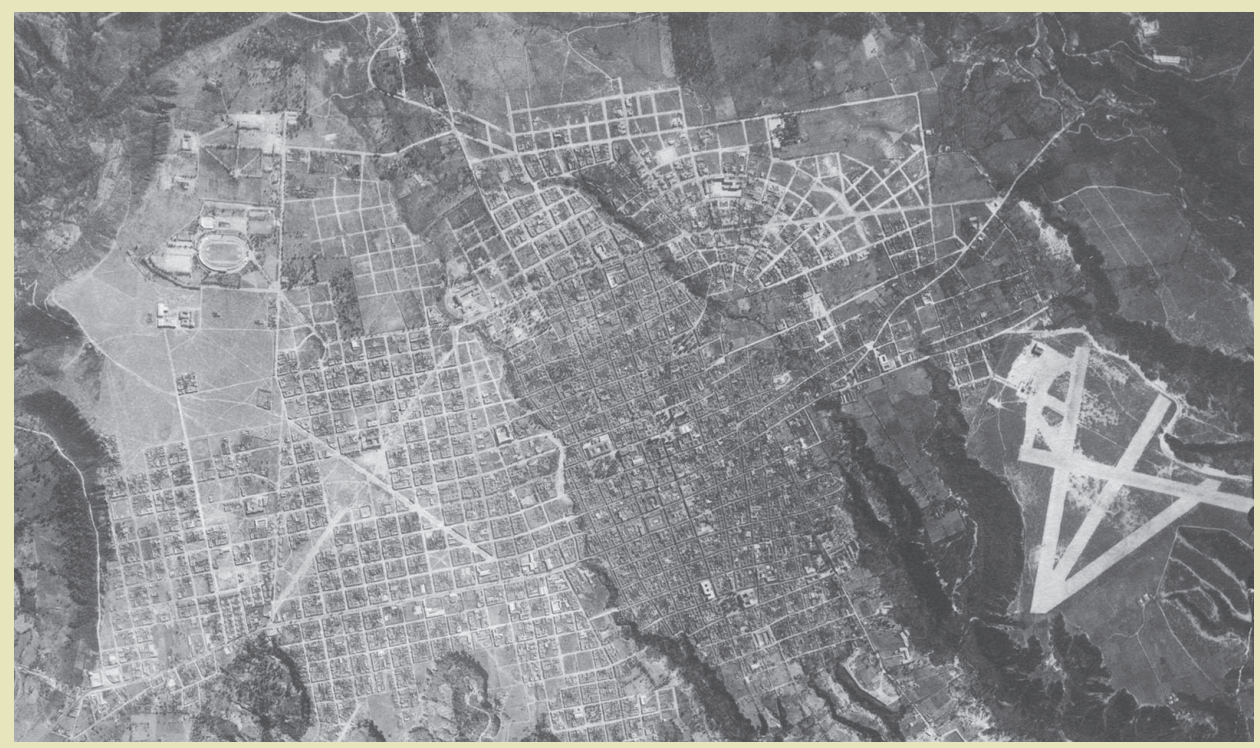

Aerofotografía de Bucaramanga de 1947 (IGAC) Fuente: Archivo Laboratorio de Investigaciones Urbanas LIU, Facultad de Arquitectura Universidad Santo Tomás, Bucaramanga. Copia suministrada por el autor

\begin{abstract}
This article shows the impact produced by the enterprice "Robledo Hermanos" in the urbanization process of Bucaramanga during the 40's and the 70's of the twentieth century. The article is organized according to the following structure: First an introduction which briefly describes how the firm "Robledo Hermanos" settled in Bucaramanga and started its urbanizating journey, accompanied by a succint historical contextualization. Then some of the Synthesis results of the urbanistical operations developed by Robledo Hermanos along thirty years. Finally some conclusions that may allow the rise of future researches are showed.
\end{abstract}

\section{KEYWORDS}

Bucaramanga, Robledo Hermanos, Urbanization, Urban actors 


\section{INTRODUCCIÓN}

\section{El origen de la firma}

La firma Robledo Hermanos de estirpe antioqueña, tuvo su origen en una familia de Ingenieros y empresarios que decidieron asentarse y probar suerte en el oriente colombiano, tras la profunda crisis que vivía Medellín a comienzos de la década del treinta del siglo XX. Es así como los Ingenieros Fabio y Elías Robledo Uribe, junto con el Ingeniero Rafael Jaramillo, deciden buscar suerte en la ciudad de Cúcuta, a quienes posteriormente se unirían sus hermanos Iván, y Diego Robledo. Allí trabaron amistad con don Jorge Enrique Barco y Doña Julieta Vargas de Barco, padres del Ex presidente Virgilio Barco Vargas y también con el Alemán Fritz Larsen con quien se asociaron.

Llevaron a Cúcuta la representación de la industria de Hilados y Tejidos del Hato que daría origen a FABRICATO en la ciudad de Medellín, así mismo, la representación de Talleres Robledo, con origen en esta misma ciudad. Fundaron la firma Robledo y Jaramillo, dedicada a vender telas, tejidos y relojes para las torres de las iglesias a varios pueblos del Norte de Santander y también a "medir lotes y fincas".

Como los negocios prosperaban Iván, quien practicaba tenis en el Club Cazadores, trabó amistad con la familia Larsen, con quienes más tarde fundaría la firma Larsen y Robledo. La familia Larsen tenía vínculos con Europa y poseía la representación de la Casa Lister, pero debido a los conflictos que presagiaban la Segunda Guerra Mundial y las presiones sobre la familia Larsen, de común acuerdo resolvieron que se liquidaba la firma y Fabio Robledo compró su participación a su socio Larsen, así se transformó en Robledo Hermanos en Cúcuta en 1935.

Fabio Robledo llegó a Bucaramanga ese mismo año, estimulado por la posibilidad de expandir los negocios de la firma, dedicada básicamente al comercio y a las representaciones que habían adquirido del señor Larsen. Abrieron en Bucaramanga un almacén en el Pasaje Cadena, mientras Iván se quedó en Cúcuta. La casa matriz de Cúcuta financiaba la sucursal de Bucaramanga, pero los negocios en la capital santandereana fueron prosperando más rápidamente y con el tiempo decidieron cerrar Cúcuta y radicarse en Bucaramanga.

La aparición de las oportunidades inmobiliarias en Bucaramanga, propiciaron la incorporación del Ingeniero Elías Robledo, formado en la Escuela Nacional de Minas. Ello configuraba un equipo de ingenieros, arquitectos y comerciantes que comenzaban a visionar los desarrollos urbanos de la ciudad de Bucaramanga. A partir de allí, se combinaron las actividades comerciales con las urbanísticas, donde su primer escenario sería el norte de la ciudad.

Alfonso Restrepo Mejía se unió a la Firma y con el tiempo llegó a ser el Gerente de la sucursal de Bucaramanga, su amistad con don Ezequiel Alarcón produjo la Sociedad Restrepo \& Alarcón, a la que posteriormente se incorporó Robledo Hermanos y se creó Robledo Alarcón \& Restrepo Ltda., (R.A.R.O Ltda.) Esta firma se encargaba de la compra de las tierras, mientras que Robledo Hermanos urbanizaba y construía. Otras sociedades creadas por la familia Robledo fueron: Ganadería Villabel Ltda., Robledo, Tristancho \& CIA Ltda. (R.OT.O.), Provenza Ltda., Industrial de Construcciones Ltda. Sobre la dinámica de estas firmas se dará cuenta a lo largo del desarrollo del artículo. 


\section{El contexto de la ciudad}

A finales de la década del treinta, la ciudad de Bucaramanga se acercaba a los 50.000 habitantes. Su desarrollo urbano era una realidad, el crecimiento por extensión continua' entre las quebradas Seca y la Rosita, en dirección este, y los proyectos urbanizadores iniciados a mediados de la década del veinte: La Mutualidad al norte, Sotomayor al Oriente y el proceso urbanizador orgánico en las inmediaciones de la Concordia a lo largo de la calle Charco Largo, se convertían en aliciente para las nuevas campañas urbanizadoras.

La modalidad del urbanizador "clásico" ${ }^{2}$ fue empleada de forma sistemática a lo largo de las décadas del veinte hasta la del setenta. Mediante esta modalidad, los proyectos iniciados de forma anticipada y ya mencionados arriba, se convertirán en los eslabones que servirán para el arranque de las primeras iniciativas urbanizadoras de la firma.

El periodo comprendido entre mediados de la década del treinta y comienzos de los cuarenta estuvo marcado por algunos fenómenos importantes que impulsaron el desarrollo de la ciudad y dieron vigor a las iniciativas privadas urbanizadoras, entre ellas la realización de los Juegos Nacionales que se llevarían a cabo en 194I. Estas justas deportivas motivaron la modernización de la ciudad, obligó a la reubicación del aeropuerto, la construcción de los escenarios deportivos, el mejoramiento de la infraestructura vial, la adecuación de equipamientos, hotelería y todo lo necesario para atender a los deportistas.

A partir de la década del cuarenta la actividad industrial de mediana escala comenzará a formar parte del paisaje urbano, especialmente sobre la meseta de Bucaramanga y algunos reductos del norte, sobre la vía a la estación del ferrocarril. Ello propició la migración a la ciudad de una población de estratos básicamente populares que debía ser albergada. Este hecho estimuló a los urbanizadores privados como Robledo Hermanos a introducirse de forma decidida en la creación de nuevos barrios, que cubrirían estos segmentos de población, proceso que se ha denominado para esta investigación como Proyectos de borde, la sintonía con la vivienda popular.

Pero la clase media y alta conformada especialmente por industriales, comerciantes y empleados demandaba también nuevas urbanizaciones, que los urbanizadores privados abordaron decididamente, en el caso de Robledo Hermanos este segmento de mercado se ha denominado: proyectos del este: entre la clase media y la élite local. A finales de la década del sesenta comenzó el proceso de expansión urbana hacia el sur de la ciudad, entre Bucaramanga y Floridablanca, generándose nuevos suelos urbanizados de carácter periférico que se denominaron como: la colonización del sur: la apuesta por la nueva ciudad y la alianza público privada. En el marco de este escenario se produjeron los proyectos que se describen a continuación.

I Para tener una visión amplia de este proceso, ver: RUEDA GÓMEZ, Néstor José. La formación del área metropolitana de Bucaramanga: el papel de la vivienda del Instituto de Crédito Territorial como elemento clave de su configuración. Universidad Politécnica de Valencia - Departamento de Urbanismo, Valencia. 2012. Tesis doctoral inédita.

2 "Las urbanizaciones son operaciones de suelo. Se pretende únicamente parcelar unos terrenos de superficie variable para, con un rendimiento máximo, establecer los lotes edificables y venderlos. (...) este proceso es conducido por un único promotor, persona física o jurídica, que previamente ha adquirido la propiedad del suelo y que monopoliza todo lo que se refiere a la producción del suelo parcelado.” GIMÉNEZ BALDRÉS, Enrique J. Parcelaciones residenciales suburbanas. La formación de la periferia metropolitana de Valencia. Valencia, Universidad Politécnica, 1996. P. 595 
Tabla I. Número y porcentaje de urbanizaciones y lotes por escenario. Fuente: elaboración del autor a partir del análisis de las urbanizaciones

\section{Breve descripción y análisis general de los proyectos}

\begin{tabular}{|c|c|c|c|c|}
\hline \multicolumn{5}{|c|}{ NÚMERO Y PORCENTAJE DE URBANIZACIONES Y PARCELAS POR ESCENARIO } \\
\hline Urbanizaciones de Borde & $\mathrm{P} \times \mathrm{U}$ & Total Par. & Total Urb. & $\% \mathrm{P} \times \mathrm{U}$ \\
\hline Navas & 100 & \multirow{10}{*}{1957} & \multirow{10}{*}{10} & \multirow{10}{*}{24} \\
\hline Barrio Norte Suarez & 288 & & & \\
\hline Campo Hermoso & 50 & & & \\
\hline San Miguel & 700 & & & \\
\hline Parque Romero & 80 & & & \\
\hline Ricaurte & 198 & & & \\
\hline Barrio Norte & 208 & & & \\
\hline Comunero & 40 & & & \\
\hline García Rovira & 45 & & & \\
\hline Barrio Nuevo & 243 & & & \\
\hline \multicolumn{2}{|l|}{ Urbanizaciones del Este } & \multirow{8}{*}{1997} & & \\
\hline La Sevillana & 120 & & \multirow{7}{*}{7} & \multirow{7}{*}{24} \\
\hline Parque de los Niños & 18 & & & \\
\hline Antiguo Campestre & 212 & & & \\
\hline Conucos & 27 & & & \\
\hline Sotomayor & 980 & & & \\
\hline Alarcón & 312 & & & \\
\hline San Alonso & 328 & & & \\
\hline \multicolumn{2}{|l|}{ Urbanizaciones de la Periferia Sur } & & & \\
\hline Villabel & 2430 & \multirow{2}{*}{4350} & \multirow{2}{*}{2} & \multirow{2}{*}{52} \\
\hline Provenza & 1920 & & & \\
\hline TOTALES & & 8304 & 19 & 100 \\
\hline
\end{tabular}

En la Tabla I se ordenan las principales urbanizaciones desarrolladas y el número de lotes en los diferentes escenarios. Se puede observar que en las urbanizaciones de borde eran en general actuaciones urbanas de pequeño tamaño, salvo San Miguel, que alcanzó el $40 \%$ del total de las nueve restantes, con 700 lotes. En tanto que en el Este, es Sotomayor el que marca la pauta con el $49 \%$ con 980 lotes; por su parte, Alarcón y San Alonso fueron importantes actuaciones que jalonaron el desarrollo del nororiente de la meseta.

Las dos urbanizaciones ubicadas sobre la periferia sur, Villabel y Provenza fueron las únicas actuaciones de Robledo Hermanos entre Bucaramanga y Floridablanca, pero su importancia fue capital, sólo estas suman el $52 \%$ de todos los lotes vendidos de la firma en toda su historia.

La Tabla I permite una lectura de los lotes iniciales de las actuaciones, número de proyectos y el peso en términos de porcentaje que tuvieron por cada escenario analizado. Algo que es más claro cuando se relacionan los proyectos en el plano. La localización de los proyectos la realizó la propia firma sobre un plano de la ciudad de 1953.

Figura I. Plano de 1953 y la localización de los proyectos desarrollados por la firma sobre la Meseta de Bucaramanga. Fuente: Fondo Robledo Hermanos. Laboratorio de Investigaciones Urbanas LIU, Facultad de Arquitectura, Universidad Santo Tomás de Bucaramanga. Copia suministrada por el autor

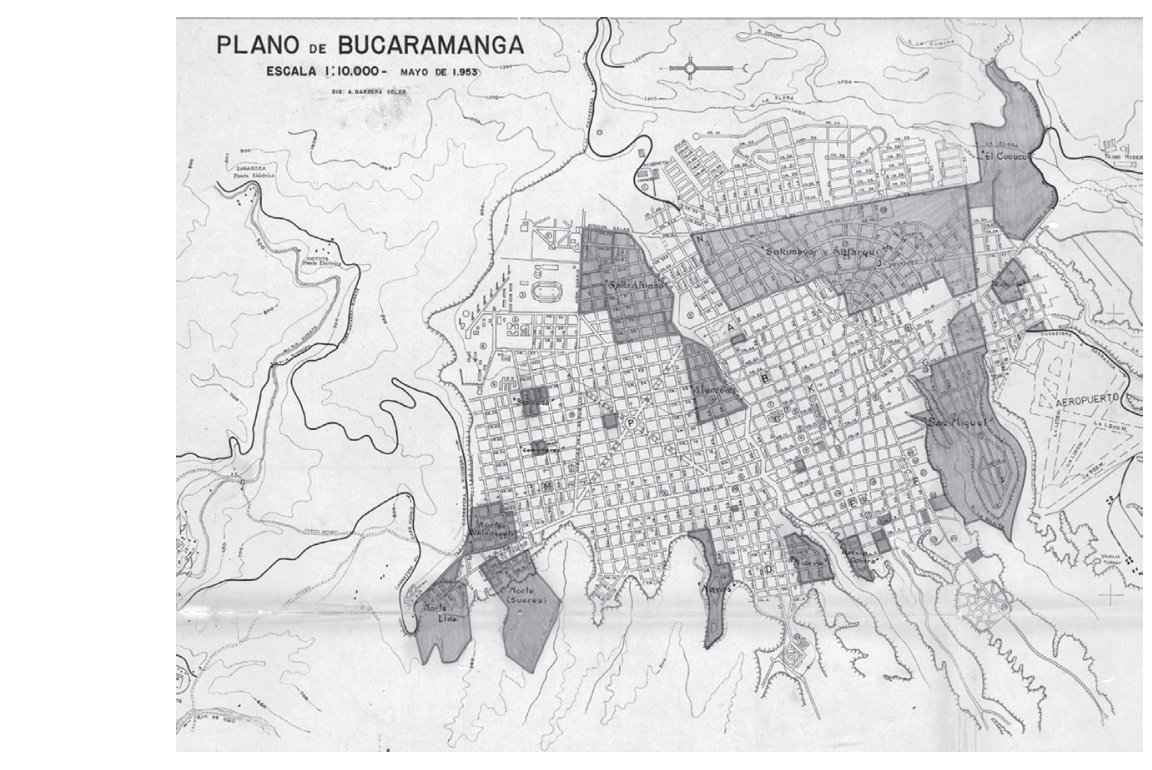

REVISTA M VOL. 9 No.2. JULIO-DICIEMBRE 2012 • FACULTAD DE ARQUITECTURA • UNIVERSIDAD SANTO TOMÁs COLOMBIA M 100 


\section{La periodización de las urbanizaciones}

El arranque ${ }^{3}$ de las urbanizaciones de la Firma Robledo se puede caracterizar en tres periodos, el vínculo con la élite local años cuarenta; las actuaciones de borde y de intersticios años cincuenta y los proyectos de periferia años sesenta.

Aunque en la década del cuarenta sólo se producen tres urbanizaciones, es incuestionable la importancia que tuvieron para la consolidación de la meseta. Sotomayor, no fue un proyecto original de Robledo Hermanos, pero sí quien planteó su estrategia de mercado y terminó desarrollándolo. Alarcón por su parte, fue el asiento de una buena parte de la élite comercial de la ciudad y en suelos de uno de sus aliados Don Ezequiel Alarcón, con quien fundarían la firma Robledo, Alarcón \& Restrepo Ltda. En el caso de San Alonso, su situación entre el escenario dotacional más importante de la meseta, sobre el que gravita la Unidad Deportiva Alfonso López, la Quinta Brigada y el batallón Caldas, así como las dotaciones educativas universitarias como la Universidad Industrial de Santander y establecimientos de secundaria, lo mismo que el Hospital Ramón González Valencia y la Facultad de Salud al sur garantizaron su éxito inmobiliario.

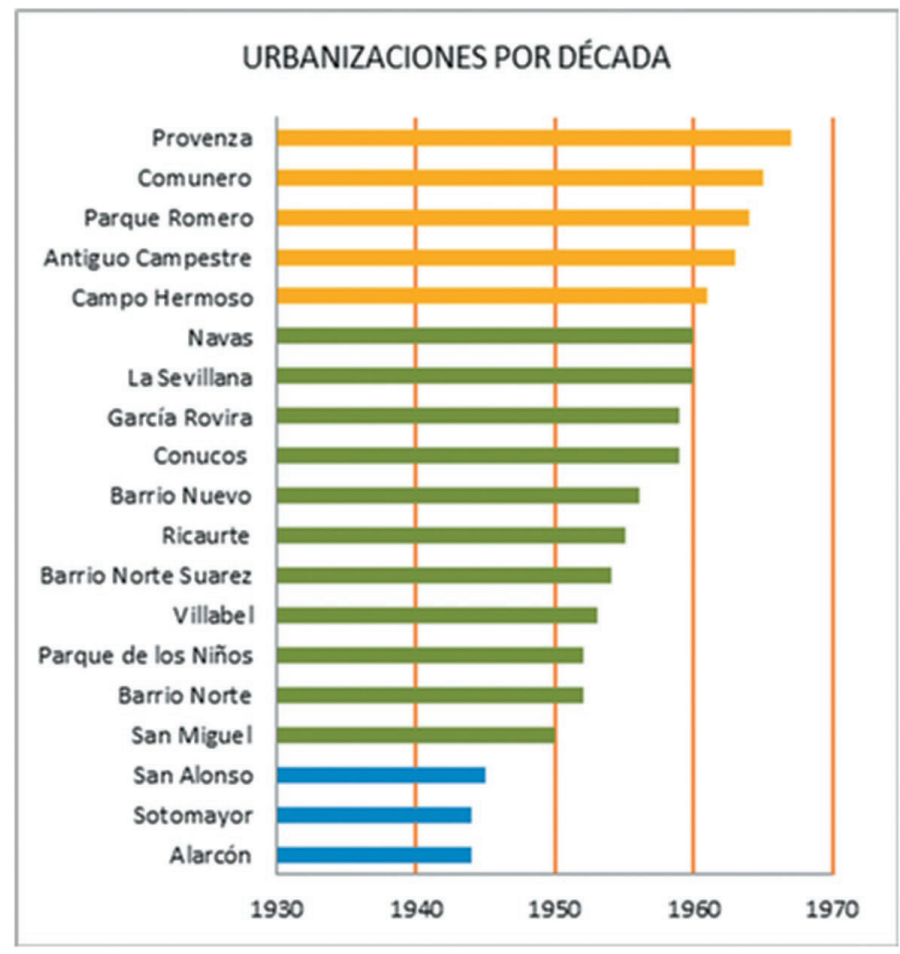

Como se aprecia en la Tabla 2, la década del cincuenta fue la más prolífica de la firma, periodo de plena consolidación de la meseta de Bucaramanga y en la que la urbanizadora participa de forma importante con actuaciones como la de San Miguel y la consolidación definitiva de Sotomayor; las demás urbanizaciones tuvieron menos impacto que las anteriores. Este periodo es también el punto de inflexión de las operaciones urbanizadoras sobre la periferia sur, con Villabel, algo que servirá tanto a los promotores privados como a la promoción pública, para decidirse por los suelos del sur, más baratos y con un clima que sirvió de estímulo a todos por igual.

3 Se define el "arranque", como el momento en el que el urbanizador inicia el proceso de urbanización y parcelación, dado que su consolidación edilicia es el producto de un largo proceso, que suele durar hasta veinte años. Aquí cabe aplicar el modelo teórico que Manuel Sola Morales, definió para explicar las formas de crecimiento de la ciudad.
Tabla 2. Urbanizaciones por década. Fuente: Elaboración del autor a partir del análisis de las urbanizaciones 
Figura 2. Modelo teórico de las formas de crecimiento urbano. Fuente: SOLÁ MORALES i Rubio Manuel, Las formas de crecimiento urbano. Ediciones UPC, Barcelona, 1997. Copia suministrada por el autor
A lo largo de la década del sesenta se inician cinco proyectos de los cuales el más importante será sin duda Provenza, que llegará para reforzar y garantizar las actuaciones de la firma al sur, pero en este caso sobre el eje de la autopista. Algo que impondrá un plus urbanizador que estimulará otros proyectos privados y el emplazamiento de instituciones educativas como el INEM sobre su costado occidental. El otro proyecto que vale mencionar es el Antiguo Campestre, urbanizado sobre los predios del antiguo Club Campestre, ya trasladado entre el Río Frio y la Quebrada Zapamanga en Floridablanca. Las otras tres urbanizaciones, fueron discretas actuaciones irrelevantes.

Desde este modelo es aplicable, en forma general, el Ensanche, aunque con la salvedad, que sólo es posible para comprender su temporalidad y sus etapas, es decir, el urbanizador parcela sobre planos, urbaniza una pieza determinada y posteriormente edifica. Una acción que, por lo general, se hace de forma individual. Este modelo de alguna manera es adaptable a los proyectos desarrollados por Robledo Hermanos sobre la meseta.

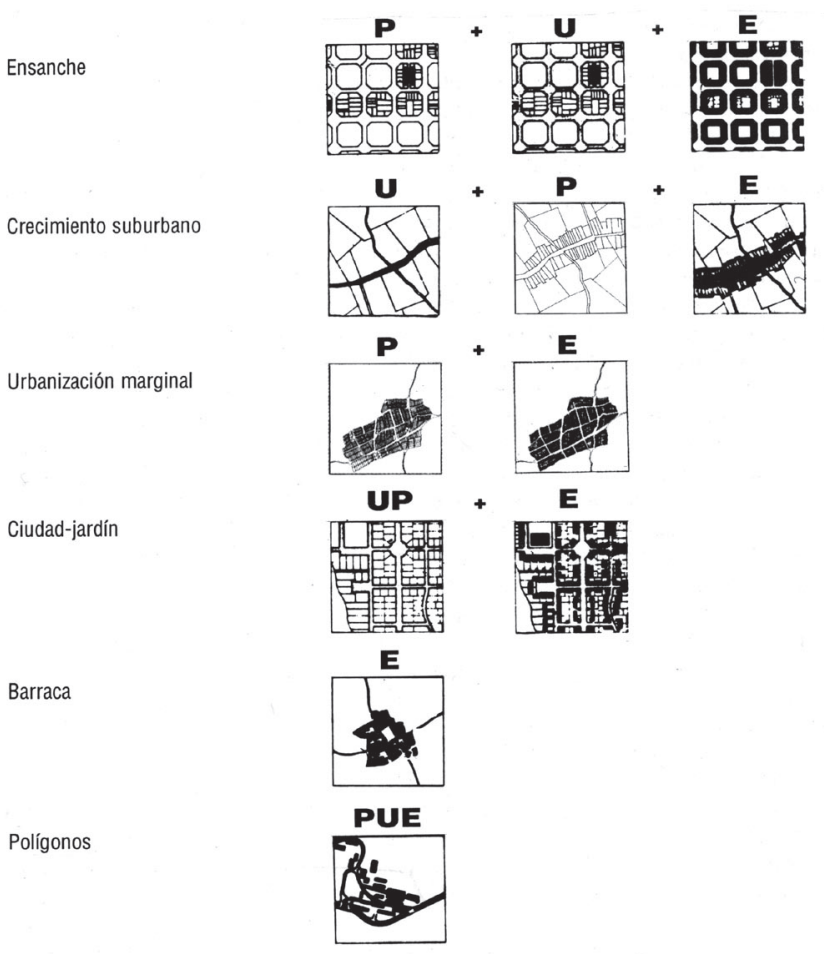

\section{$\mathbf{P}=$ Parcelación \\ U = Urbanización \\ $E=$ Edificación}

En el caso de los proyectos Villabel y Provenza, se asimila más al modelo de Crecimiento suburbano, por cuanto su forma de operación varía sustancialmente, en este sentido se asemeja a la caracterización que E. Giménez hace del urbanizador: 
"Las urbanizaciones son operaciones de suelo. Se pretende únicamente parcelar unos terrenos de superficie variable para, con un rendimiento máximo, establecer los lotes edificables y venderlos. La vialidad no tiene, por ello, otra función que la de asegurar la accesibilidad y delimitar las manzanas parcelables. Este proceso es conducido por un único promotor, persona física o jurídica, que previamente ha adquirido la propiedad del suelo y que monopoliza todo lo que se refiere a la producción de suelo parcelado."4

\begin{tabular}{|c|c|c|c|c|c|c|}
\hline \multicolumn{7}{|c|}{ PROYECTOS ROBLEDO HERMANOS } \\
\hline No. & PROYECTOS & AÑo & Década & $\begin{array}{c}\text { Proyectos por } \\
\text { década }\end{array}$ & $\begin{array}{l}\text { Total lotes } \\
\text { por década }\end{array}$ & $\begin{array}{c}\text { \% lotes por } \\
\text { década }\end{array}$ \\
\hline 1 & Alarcón & 1944 & \multirow{3}{*}{40} & \multirow{3}{*}{3} & \multirow{3}{*}{1620} & \multirow{3}{*}{20} \\
\hline 2 & Sotomayor & 1944 & & & & \\
\hline 3 & San Alonso & 1945 & & & & \\
\hline 4 & San Miguel & 1950 & \multirow{9}{*}{50} & \multirow{9}{*}{9} & \multirow{9}{*}{4157} & \multirow{9}{*}{50} \\
\hline 5 & Barrio Norte & 1952 & & & & \\
\hline 6 & Parque de los Niños & 1952 & & & & \\
\hline 7 & Villabel & 1953 & & & & \\
\hline 8 & Barrio Norte Suarez & 1954 & & & & \\
\hline 9 & Ricaurte & 1955 & & & & \\
\hline 10 & Barrio Nuevo & 1956 & & & & \\
\hline 11 & García Rovira & 1959 & & & & \\
\hline 12 & Conucos & 1959 & & & & \\
\hline 13 & La Sevillana & 1960 & \multirow{7}{*}{60} & \multirow{7}{*}{7} & \multirow{7}{*}{2523} & \multirow{7}{*}{30} \\
\hline 14 & Navas & 1960 & & & & \\
\hline 15 & Campo Hermoso & 1961 & & & & \\
\hline 16 & Antiguo Campestre & 1963 & & & & \\
\hline 17 & Parque Romero & 1964 & & & & \\
\hline 18 & Comunero & 1965 & & & & \\
\hline 19 & Provenza & 1967 & & & & \\
\hline
\end{tabular}

Dado que en este caso el urbanizador generaba básicamente lotes y no casas, es importante analizarlos de forma cuantitativa. Una primera variable es la temporal. En la década del cuarenta se producen sólo tres actuaciones, que varían sustancialmente de escala Alarcón y San Alonso con 300 lotes en promedio y Sotomayor, que se había iniciado a mediados de la década del veinte ${ }^{5}$ con 980; la década del cincuenta fue el periodo de mayor cantidad de proyectos y como consecuencia de más producción de lotes, pese a que muchos de ellos eran de pequeña escala, como los casos de Conucos, Parque de los Niños o García Rovira, que en promedio no superaban los 30 lotes, compensado por actuaciones como Villabel con 2300 o San Miguel con 700. En la década del sesenta disminuye la cantidad de proyectos y también su tamaño. Sobre la Meseta se generaron tan sólo seis pequeñas actuaciones que en promedio no superaban los 100 lotes, explicable por la colmatación urbana de la meseta, donde el urbanizador aprovecha pequeñas franjas de terreno, cuyo caso más característico fue Campo Hermoso, aunque es importante destacar que para este caso Provenza contribuye con un alto porcentaje, el $76 \%$ del total de lotes en este periodo.

4 GIMÉNEZ Baldrés, Enrique. Parcelaciones Residenciales Suburbanas, la forma de la periferia metropolitana de Valencia. Ed. Generalitat de Valencia, UPV. Colegio oficial de arquitectos de Valencia. 1996

5 En 1937 en el ocaso de la década de los treinta se optó por la liquidación, labor que se encomendaría a la Firma Robledo Hermanos, con la asesoría de Don Ricardo Olano, el 23 de junio de 1937. Para ampliar este proceso ver: La Formación ver, Op. Cit. Rueda Gómez, La formación del Área Metropolitana pp. |4|-I55

Tabla 3. Síntesis de proyectos realizados por la firma Robledo Hermanos en Bucaramanga Fuente: Elaboración del autor 


\section{La manzana como elemento diferenciador}

Las manzanas son el elemento diferenciador más característico de estas urbanizaciones. En los diferentes proyectos desarrollados se pudieron identificar tres tipologías de manzana: reticular regular, reticular irregular y manzana en hilera.

Tabla 4. Tipologías de Manzana. Fuente: Elaboración del autor a partir del análisis de las urbanizaciones
Figuras 3 A y 3B. Tipologías de Manzana. Reticular Regular, Reticular Irregular y en Hilera. Fuente: elaboración del autor a partir del análisis de las urbanizaciones. En la Figura 3 A se observa en la parte central la primera tipología (Reticular Regular) y sobre el borde inferior, se observa lo segunda tipología (Reticular Irregular). En la Figura 3B se observa la tipología de Hilera

\begin{tabular}{|l|c|}
\hline \multicolumn{2}{|c|}{ Tipos de manzana según proyecto } \\
\hline \multicolumn{1}{|c|}{ URBANIZACIÓN } & MORFOLOGíA DE MANZANA \\
\hline Parque de los Niños & Reticular irregular \\
\hline García Rovira & Reticular regular \\
\hline Conucos & Hilera \\
\hline Comunero & Reticular regular \\
\hline Parque Romero & Hilera \\
\hline Campo Hermoso & Hilera \\
\hline Navas & Reticular irregular \\
\hline La Sevillana & Hilera \\
\hline Antiguo Campestre & Hilera \\
\hline Ricaurte & Reticular regular \\
\hline Barrio Norte & Reticular regular \\
\hline Barrio Nuevo & Reticular regular \\
\hline Barrio Norte Suarez & Hilera \\
\hline San Miguel & Reticular irregular \\
\hline Alarcón & Reticular regular \\
\hline San Alonso & Hilera \\
\hline Provenza & Reticular irregular \\
\hline Sotomayor & Hilera \\
\hline Villabel & \\
\hline
\end{tabular}

En las imágenes que se referencia a continuación se pueden observar las distintas morfologías clasificadas
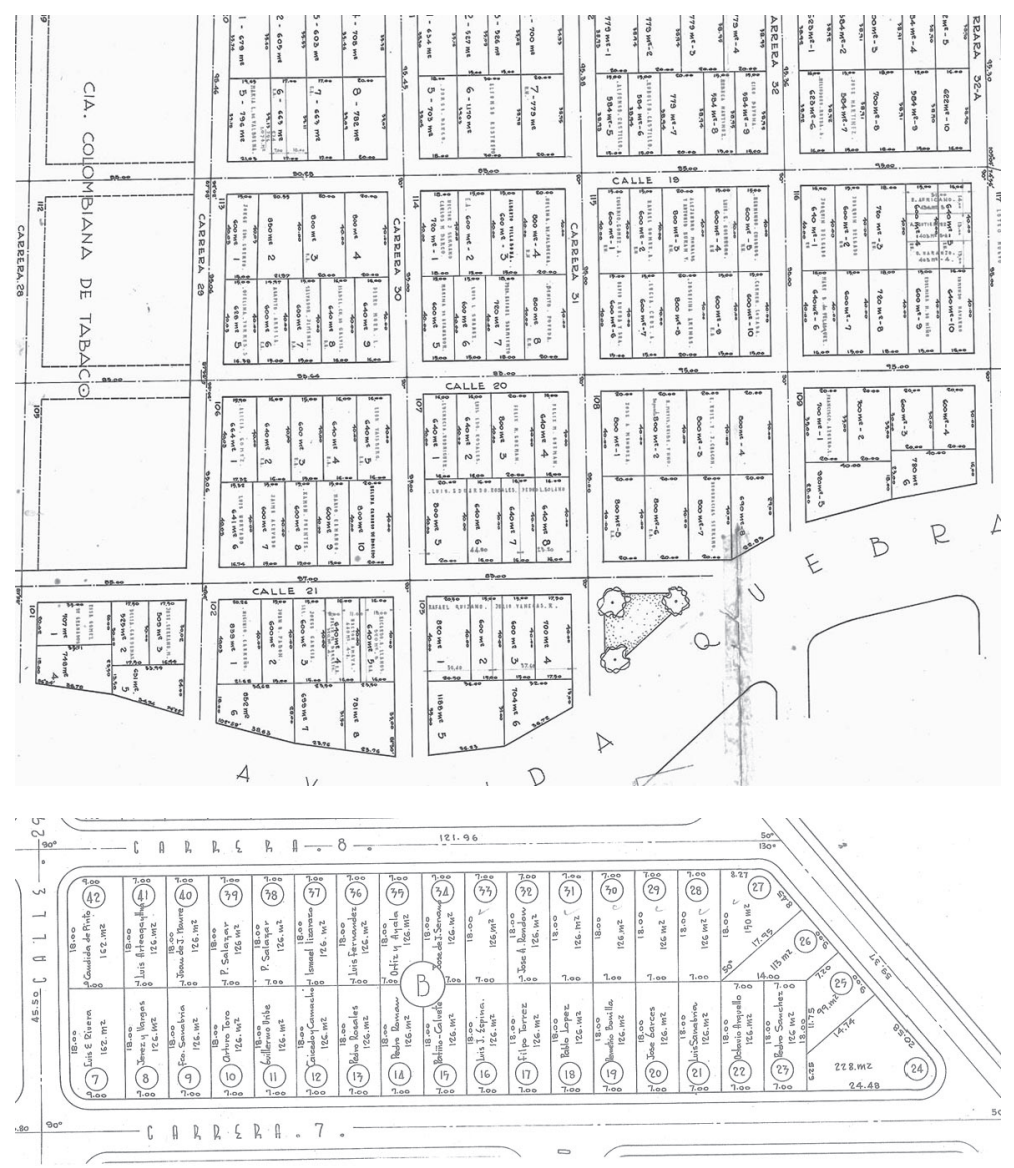
Las tipologías son indicativas de la época en la que se producen los proyectos. En el caso de la retícula regular, se origina por el amoldamiento de la continuidad de la trama urbana preexistente sobre la meseta de Bucaramanga; en el caso de la retícula irregular está condicionada básicamente por las condiciones topográficas, generalmente sobre suelos de escarpa o sobre el borde de las quebradas. Estas se corresponden en casi su totalidad a la década del cuarenta y las restantes al cincuenta, a partir de la década del sesenta, la tipología en hilera es la característica predominante de las urbanizaciones, su aplicación de tendencias racionalistas marca la pauta, como ocurrió en San Miguel, donde la "optimización" del suelo llegó a densidades hasta de $47 \mathrm{v} / \mathrm{Ha}$. Tendencia que se mantiene en la década del setenta en Provenza con predios en promedio de $240 \mathrm{~m}^{2}$ por parcela para ambos casos.

\section{La relación del impacto de las actuaciones Robledo Hermanos vs. ICT entre 1940 y 1970}

Una manera de poder medir el peso que tiene un urbanizador en la producción de suelo urbano, es compararlo con otros en el mismo periodo estudiado, bien sea público o privado. Para este caso particular se comparó a Robledo Hermanos y el Instituto de Crédito Territorial, (ICT) por cuanto se disponía de datos confiables. En la Tabla 5 se muestra la síntesis de los dos promotores a lo largo de las tres décadas analizadas.

\begin{tabular}{|c|c|c|c|c|c|c|c|c|c|}
\hline PROYECTOS R. H. & Década & $\begin{array}{l}\text { Proyectos } \\
\text { por década }\end{array}$ & $\begin{array}{c}\text { Total } \\
\text { lotes por } \\
\text { década }\end{array}$ & $\begin{array}{c}\% \text { lotes } \\
\text { por década }\end{array}$ & Proyectos Ict & Década & $\begin{array}{l}\text { Proyectos } \\
\text { por década }\end{array}$ & \begin{tabular}{|c|} 
Total \\
viviendas por \\
década
\end{tabular} & $\begin{array}{l}\% \text { viviendas } \\
\text { por década }\end{array}$ \\
\hline Alarcón & \multirow{3}{*}{40} & \multirow{3}{*}{3} & \multirow{3}{*}{1620} & \multirow{3}{*}{20} & \multirow{3}{*}{ La Aurora } & \multirow{3}{*}{40} & \multirow{3}{*}{1} & \multirow{3}{*}{142} & \multirow{3}{*}{2} \\
\hline Sotomayor & & & & & & & & & \\
\hline San Alonso & & & & & & & & & \\
\hline San Miguel & \multirow{9}{*}{50} & \multirow{9}{*}{9} & \multirow{9}{*}{4157} & \multirow{9}{*}{50} & \multirow{3}{*}{ Modelo } & \multirow{9}{*}{50} & \multirow{9}{*}{9} & \multirow{9}{*}{1002} & \multirow{9}{*}{15} \\
\hline Barrio Norte & & & & & & & & & \\
\hline Parque de los Niños & & & & & & & & & \\
\hline Villabel & & & & & \multirow{2}{*}{ Álvarez Restrepo } & & & & \\
\hline Barrio Norte Suárez & & & & & & & & & \\
\hline Ricaurte & & & & & \multirow{2}{*}{ Pan de Azúcar } & & & & \\
\hline Barrio Nuevo & & & & & & & & & \\
\hline García Rovira & & & & & \multirow{2}{*}{ La Victoria } & & & & \\
\hline Conucos & & & & & & & & & \\
\hline La Sevillana & \multirow{13}{*}{60} & \multirow{13}{*}{7} & \multirow{13}{*}{2523} & \multirow{13}{*}{30} & Campo Hermoso & \multirow{16}{*}{60} & \multirow{16}{*}{16} & \multirow{16}{*}{5737} & \multirow{16}{*}{83} \\
\hline \multirow{2}{*}{ Navas } & & & & & La Joya & & & & \\
\hline & & & & & Terrazas & & & & \\
\hline Campo Hermos & & & & & Puerta Del Sol & & & & \\
\hline 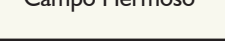 & & & & & La Ceiba & & & & \\
\hline & & & & & San Alonso & & & & \\
\hline Antiguo Campestre & & & & & $\begin{array}{c}\text { La Salle } \\
\text { Min. Hacienda }\end{array}$ & & & & \\
\hline Parque Romero & & & & & Mutis & & & & \\
\hline 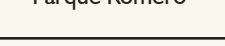 & & & & & Santander & & & & \\
\hline Comuneros & & & & & Antonia Santos & & & & \\
\hline evinuter os & & & & & Villabel & & & & \\
\hline Provenza & & & & & Los Lagos & & & & \\
\hline Provenza & & & & & Los Lagos & & & & \\
\hline & & & & & Kennedy & & & & \\
\hline & & & & & San Cristóbal I Y II Etapa & & & & \\
\hline & & & & & La Juventud & & & & \\
\hline
\end{tabular}


Figura 4. Lotes proyectados por Robledo Hermanos e ICT entre 1940 y 1970
A partir de los datos obtenidos, se pueden concluir varios asuntos importantes: en primer lugar, que ante la ausencia del Estado como promotor inmobiliario, se produjo una acción decidida de los urbanizadores privados para generar suelo urbano. En la década cuarenta esta dinámica es clara y se mantiene en la siguiente, aunque ya haya aparecido el ICT; la capacidad política y económica el Estado a través del Instituto fue contundente entre 1960 y 1970, en el que el ICT produjo el 39\% más viviendas que los lotes de Robledo Hermanos. En la Figura 4 se puede ver con claridad la dinámica comparada del desarrollo de los proyectos por década, en términos porcentuales.

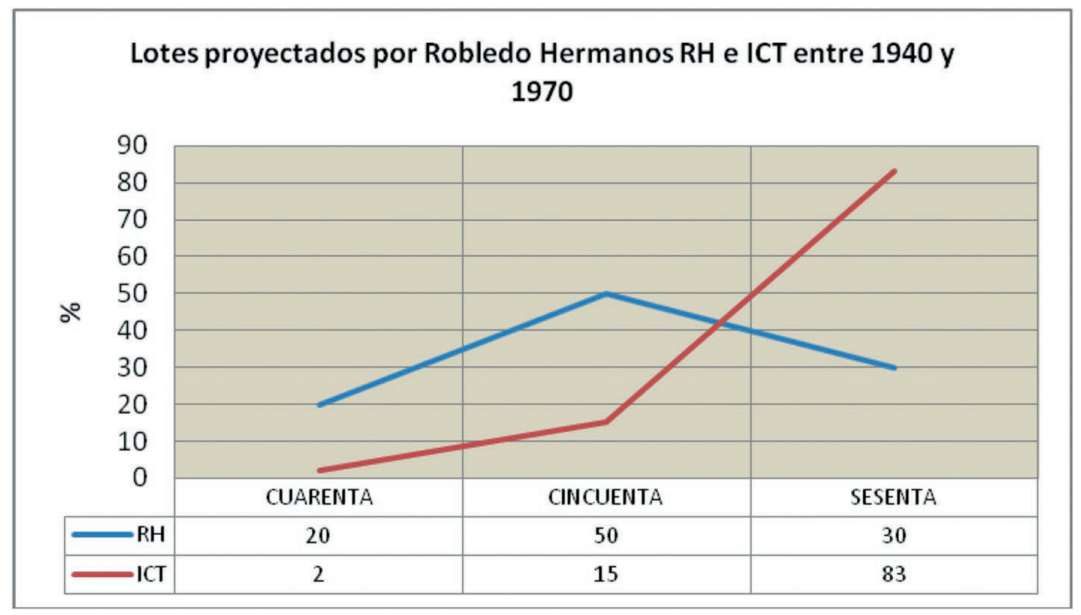

Fuente: elaboración del autor a partir de datos de la investigación

Un aspecto adicional que puede ser indicativo para esclarecer el declive de la firma Robledo Hermanos, tiene que ver con la competencia de un mismo mercado inmobiliario con el ICT. Si bien en la década del cuarenta Robledo Hermanos había trabajado para un segmento de mercado de clase media y alta, en las subsiguientes se centró en un mercado más popular, como puede evidenciarse en el plano general de localización de los proyectos. En este se ve claramente que el ICT urbaniza sobre las franjas de filamentos en una franca competencia en la que el ICT ganará la partida, por cuanto sus proyectos contaban acompañados de subsidios, una organización técnica, financiera y política que garantizaba a los adjudicatarios no sólo el lote sino también la vivienda.

Este aspecto fue crucial para definir finalmente la partida en un mercado donde Robledo Hermanos, ante esta realidad, plantea como estrategia las alianzas para el desarrollo de proyectos de cuyos ejemplos más importantes fueron Provenza y Villabel, donde opta por vender al ICT franjas importantes de terreno para producir vivienda popular. (Ver Gráfico 4. Síntesis Proyectos Robledo Hermanos vs. ICT)

\section{CONCLUSIONES}

Derivado de esta investigación se pueden sacar varias conclusiones que pueden servir de punto de partida para investigaciones venideras. 
En primer lugar es importante decir, que Robledo Hermanos fue un promotor inmobiliario de carácter privado, que llegó a la ciudad en un periodo de crisis generalizada y que fue esta misma la que propició su incorporación a la dinámica urbanizadora tras responsabilizarse de la liquidación de la Compañía de Fomento Urbano de Santander, una investigación pormenorizada de los aspectos económicos que guiaban el mercado inmobiliario y sus dinámicas, bien podría representar un ejercicio valioso.

Así mismo, se pueden caracterizar tres periodos de su proceso urbanizador: el primero, la ligazón de la Familia Robledo con la élite local, que le valieron múltiples alianzas que definieron este primer periodo con proyectos para clase media y alta, década del cuarenta; un segundo periodo, en el que se advierten intervenciones de pequeña escala para segmentos de población de estratos bajos, década del cincuenta; y finalmente un tercer periodo caracterizado por el desarrollo de proyectos periféricos al sur en alianza con el ICT y algunas discretas actuaciones dentro del suelo urbano consolidado. Cada periodo podría ser indicativo de la forma como los urbanizadores leían los segmentos de mercado y los intereses económicos de los promotores privados.

Por último, se evidencia el impacto que produce la intervención pública dentro de un segmento de mercado poco solvente que compraba lotes y que ahora podría comprar la vivienda completa y que adicionalmente estaba soportado por el aparato financiero del Estado. En estas condiciones, el repliegue de este tipo de urbanizador "clásico" se hizo evidente. Sería importante en un ejercicio posterior, investigar en paralelo otros urbanizadores con mayor éxito, que supieron capotear el temporal, para sobrevivir hasta los tiempos actuales, en casos como Urbanas, para citar uno.

\section{BIBLIOGRAFÍA}

FONDO ROBLEDO HERMANOS. Laboratorio de Investigaciones Urbanas, Facultad de Arquitectura. Universidad Santo Tomás, Bucaramanga.

GIMÉNEZ BALDRÉS, Enrique J. Parcelaciones residenciales suburbanas. La formación de la periferia metropolitana de Valencia. Valencia, Universidad Politécnica, 1996

OLANO, Ricardo. Memorias Tomo II. Colección cielos de arena, Ed. EAFIT. 2004

RUEDA GÓMEZ, Néstor José. La formación del área metropolitana de Bucaramanga: el papel de la vivienda del Instituto de Crédito Territorial como elemento clave de su configuración. Universidad Politécnica de Valencia - Departamento de Urbanismo, Valencia. 20I2. Tesis doctoral inédita.

SOLÁ MORALES i Rubio Manuel, Las formas de crecimiento urbano. Ediciones UPC, Barcelona, 1997

Vanguardia Liberal, Bucaramanga, 1960 